\title{
Apprendre et enseigner la grammaire aujourd'hui en français langue première. Le cas de la ponctuation en production écrite en sixième.
}

\author{
Véronique Paolacci \& Claudine Garcia-Debanc \\ IUFM de Midi-Pyrénées, Erte 46 GRIDIFE \\ Laboratoire Lordat Octogone, Toulouse II Le Mirail
}

\section{Introduction}

Les professeurs sont particulièrement démunis quand il s'agit d'aborder l'enseignement de la langue dans leur classe : leurs connaissances grammaticales sont peu assurées ( ceci même pour les professeurs de collège comme ils en témoignent souvent en formation continue). Quelle langue enseigner? Comment répondre aux besoins des élèves, surtout en situation d'écriture? Toutes ces questions sont particulièrement vives quand on aborde l'enseignement de la ponctuation. Cet enseignement se trouve aux carrefours de l'oral et de l'écrit, des usages et de la norme et concerne autant la production que la réception (écriture vs lecture). Les programmes (de l'école et du collège, 2007, 1995) citent la ponctuation comme une notion fondamentale à travailler dès le niveau CE1 (enfants de 6-7 ans). Il est également incontestable que l'enseignement de la ponctuation appartient aux " incontournables" dans les pratiques enseignantes dans le sens où à chaque niveau de leur cursus à l'école primaire, les élèves abordent au moins une fois dans l'année cet objet d'enseignement.

Notre recherche doctorale interroge à la fois les apprentissages concernant l'acte de ponctuer et l'enseignement de la ponctuation à l'école et au collège. Nous présenterons ici des illustrations de ces deux aspects, objets d'étude centraux en didactique. Dans un premier temps, l'étude d'un corpus de copies d'élèves entrant au collège permet d'envisager les compétences rédactionnelles acquises par des élèves de 11-12 ans. Quelles segmentations du texte peut-on observer dans ces textes d'élèves? Qu'apprend-on des processus mis en œuvre dans une production écrite en situation scolaire? Quelle image de la phrase graphique est illustrée dans ces écrits?

Nous aborderons ensuite le volet « enseignement ». Dans le cadre de notre thèse, nous avons observé des enseignants dans leurs pratiques quotidiennes de classe mais aussi dans des situations plus innovantes concernant l'objet d'enseignement qu'est la ponctuation. Pour répondre aux besoins des élèves illustrés dans l'étude de copies d'élèves, nous développerons dans une deuxième partie une analyse de pratiques effectives d'enseignement dans une classe de sixième selon la méthodologie d'ingénierie didactique (Artigues, 2002). Nous terminerons par un compte rendu de publications antérieures répondant à la question de la formation des enseignants. Nous montrerons ainsi en quoi les recherches en didactique du français alimentent la formation initiale et continue en IUFM et quelle place privilégiée occupent les sciences du langage parmi les disciplines contributoires.

\section{Cadre théorique}

La ponctuation est un objet d'étude complexe qui connaît différentes approches selon le champ disciplinaire concerné. Au regard des recherches linguistiques sur la question, les travaux en psychologie du langage apportent un complément précieux pour les didacticiens du français (Garcia-Debanc \& Fayol, 2001).

La ponctuation, au niveau linguistique, fait l'objet de deux tendances contrastées. D'un côté, domine une approche normative. Doppagne (1978) attribue essentiellement à la ponctuation un rôle pausal et mélodique. Dans la même perspective, Drillon (1991: 15) présente dans son ouvrage « une étude du bon 
usage qu'on fait des signes de ponctuation ». D'un autre côté, avec l'équipe de Catach (Ed., 1980), la ponctuation devient un objet linguistique à proprement parler. Catach (Ed., 1980:21) donne cette définition de la ponctuation : c'est un «ensemble des signes visuels d'organisation et de présentation accompagnant le texte écrit, intérieurs au texte et communs au manuscrit et à l'imprimé ; la ponctuation comprend plusieurs classes de signes graphiques discrets et formant système complétant et suppléant l'information alphabétique ». Les signes de ponctuation sont « dépendants et complémentaires ».

Les linguistes inventorient une liste finie des signes (plus ou moins fluctuante, il est vrai). Tournier (1980) considère quatre catégories de signes de ponctuation: la ponctuation de mot (comme le blanc entre les mots), la ponctuation de phrase (comme la virgule), la ponctuation métagraphique (comme le paragraphe) et la ponctuation spécificatrice (comme la majuscule). Védénina (1989) élargit la notion de ponctuation à ce qu'elle appelle la «présentation typographique » (étude des caractères d'imprimerie, justifications des lignes, blancs...). Anis, Chiss \& Puech (1988) font de même et classent conjointement les signes de ponctuation, les paragraphes et les blancs graphiques dans ce qu'ils nomment les « topogrammes ».

Les signes de ponctuation sont hiérarchisés selon des forces qui leur sont attribuées. Ainsi, le couple majuscule/point a une force supérieure à la virgule. De plus, les signes de ponctuation sont régis selon des lois d'usage. Catach (1994) distingue par exemple la loi de neutralisation. Quand un même ponctuant remplit plusieurs fonctions, il n'est pas répété.

Un même signe peut remplir plusieurs fonctions. Dans son ouvrage de référence, Védénina (1989) affine l'approche fonctionnelle des signes de ponctuation. Elle distingue les fonctions syntaxique, énonciative (ou communicative) et sémantique. Catach (1994) accorde également de l'importance à la fonction intonative de la ponctuation illustrée particulièrement par la modalité de la lecture à haute voix. Notons que cette fonction est largement citée par les enseignants dans leur classe. L'histoire de la ponctuation montre que lecture à haute voix et ponctuants sont étroitement liés. Les écrivains parlent également de ponctuation respiratoire. Seulement, il s'agit de ne pas oublier qu'il n'y a pas univocité entre la ponctuation et les pauses en lecture oralisée. Le paradigme de la ponctuation prend, pour ces chercheurs, la dimension d'un système polyfonctionnel dont les manuels scolaires rendent peu compte.

A partir de ces théories linguistiques, les psychologues du langage comme Schneuwly (1988), Fayol (1997) ou Favart \& Passerault (2000) se sont penchés sur les usages de la ponctuation dans des corpus écrits d'enfants et ont interrogé l'acquisition de la ponctuation. Dans une perspective développementale, les travaux en psycholinguistique renseignent sur les processus rédactionnels mis en oeuvre dans une tâche d'écriture. Grâce à des protocoles assez diversifiés (comme des tâches d'écriture sans support, des tests de closure ou des supports images), les chercheurs ont montré les liens entre ponctuation et connecteurs et le rôle privilégié joué par ces systèmes comme témoin de l'acquisition de la planification du texte. Ils ont également observé la variation de la ponctuation selon le genre d'écrit, les signes de ponctuation se diversifiant et se normalisant avec l'âge des scripteurs. Des études on line et off line ont été réalisées. Ce sont des travaux précieux pour les enseignants car ils présentent souvent des perspectives longitudinales qui manquent parfois aux professeurs enseignant parfois plusieurs années dans un même niveau de classe.

Pour Fayol (1997: 169), ponctuation et connecteurs fonctionnent comme une sorte de système de « parenthétisation ». " L'un et l'autre paradigmes tendent à regrouper/séparer des blocs de propositions ». Ils ont certes des valeurs sémantiques (trop privilégiées à une époque selon Fayol) mais marquent aussi en surface « des relations hiérarchiques entre les énoncés et pour les connecteurs, la nature de ces relations ». Dans tous les types d'écrits étudiés, le degré de liaison interpropositionnelle contraint l'occurrence des marques de ponctuation et des connecteurs. Le marquage en surface dépend d'une part de l'extension du paradigme linguistique et d'autre part, de l'utilisation plus ou moins stratégique qu'un individu peut faire des marques (Fayol, $1997: 173$ ). 
Pour résumer, deux fonctions sont attribuées au système de la ponctuation et à celui des connecteurs dans la perspective de la psychologie du langage. La principale fonction (précoce dans une perspective développementale) est « de marquer, dans le texte, le degré de relation entre les éléments tels qu'ils sont organisés au niveau de la représentation du producteur » (Passerault, $1991: 100)$. Une seconde fonction (plus tardive chez les scripteurs) est « une fonction de planification au niveau de la structure textuelle d'ensemble » (Passerault, 1991: 100). Cette fonction demande une représentation de la situation de communication.

\section{Caractérisations des difficultés d'élèves à ponctuer un texte : analyse d'un corpus de copies d'élèves de sixième}

En s'appuyant sur ces travaux, nous présentons à présent une analyse de copies d'élèves de sixième pour rendre compte des compétences rédactionnelles des élèves dans une situation d'écriture scolaire.

\subsection{Choix méthodologiques}

Notre étude est basée sur un corpus de 100 copies d'élèves de 11-12 ans issues des Evaluations Nationales à l'entrée en sixième de 2003. Les textes étudiés sont des textes narratifs et plus particulièrement des suites de textes. L'écriture des élèves est contrainte par des éléments de nature textuelle et iconographique. Il s'agit de compléter une page d'un journal fictif intitulé «Le petit collégien ». Certains éléments sont présents sur la page :

1 Un titre ( « Le trésor retrouve sa place au musée »);

2 un sous-titre ( «Quelle extraordinaire aventure pour cet enfant et son chien! »);

3 l'incipit de l'article («Dimanche dernier, en fin d'après-midi, après une longue promenade en forêt, Bob et sa famille retournaient à la voiture. Bob suivait ses parents lorsqu'il vit son chien s'engager brusquement sur un petit sentier...»);

4 un extrait d'interview de la famille en question ;

5 les dessins des personnages principaux (Bob et son chien) ainsi que le dessin du trésor dans un coffre.

Tous ces éléments caractérisent les attentes des concepteurs du sujet. Le type de texte demandé est bien un récit inscrit dans une situation d'énonciation que les élèves doivent analyser avec ce qui leur est proposé.

Nous avons réalisé un comptage des marques de ponctuation et des connecteurs utilisés par les élèves. Des rapports ont été opérés entre le nombre de ces marques d'organisation textuelle et le nombre de propositions au sein des phrases. Le comptage a été réalisé automatiquement par le logiciel TROPES pour les connecteurs. Notre classification des connecteurs dans les récits a été inspirée par celle de Favart \& Passerault (1999). Un programme a été mis au point par B. Jeunier, ingénieur de recherche à l'IUFM de Midi-Pyrénées, pour le comptage des signes de ponctuation.

\subsection{Les résultats observés}

On constate une grande hétérogénéité des compétences scripturales pour la tranche d'âge travaillée : le texte le plus court comprend 61 mots et le plus long 8 fois plus de mots soit 497 mots ; ce qui équivaut à des textes qui présentent de 12 à 70 phrases. Comme de nombreuses études l'ont montré, le point (que nous distinguons des autres types de points) et la virgule sont les signes les plus employés (voir tableau 1). Cependant, $10 \%$ des élèves observés n'utilisent pas les virgules, les points assurant alors exclusivement une fonction démarcative dans leurs textes, en les rythmant fortement, sans que soit modulée la force des ruptures. Le point est alors utilisé comme archiponctème (Fabre, 1989) surponctuant 
systématiquement les récits (Fayol, 1981) correspondant à l'utilisation de la stratégie knowledge telling décrite par Bereiter et Scardamalia (1987).

\begin{tabular}{|l|l|}
\hline Signes de ponctuation & Moyenne par copie \\
\hline Points & 11,7 \\
\hline Virgules & 5,68 \\
\hline Points d'exclamation & 2,63 \\
\hline Deux points & 1,23 \\
\hline Points d'interrogation & 0,72 \\
\hline Points de suspension & 0,31 \\
\hline
\end{tabular}

Tableau 1 : Les signes de ponctuation utilisés dans les copies de $6^{\text {ème }}$

Il est toutefois intéressant de souligner que le point d'exclamation arrive en troisième position des emplois des signes : les textes d'enfants usent de la ponctuation expressive pour ponctuer notamment les parties dialogiques, qui sont nombreuses dans les textes analysés. Dans un texte du corpus, un élève utilise 15 points d'exclamation à côté de 14 points. Quand le point d'exclamation est doublé, triplé ou même quadruplé, il acquiert pour ainsi dire une valeur logogrammique (Anis, Chiss \& Puech, 1988) comme dans cet extrait : « Bob !!! Bob !!! Disparu !!!! Chéri arrête-toi tout de suite ! » De façon générale, $70 \%$ des élèves de $6^{\text {ème }}$ observés utilisent d'autres marques que les seuls points et virgules, ce qui atteste la diversification des signes observée par Fayol (1981) à partir du CM2.

Les élèves de $6^{\text {ème }}$ emploient également l'alinéa (sous la forme soit de retours à la ligne, soit de sauts de lignes).

\begin{tabular}{|l|l|}
\hline & Pourcentages \\
\hline Textes mono-bloc & $34 \%$ (dont $19 \%$ avec des dialogues) \\
\hline Textes avec un alinéa & $14 \%$ (dont $9 \%$ avec des dialogues) \\
\hline De 2 à 8 alinéas & $23 \%$ (dont $18 \%$ avec des dialogues) \\
\hline De 9 à 40 alinéas & $29 \%$ (dont $26 \%$ avec des dialogues) \\
\hline
\end{tabular}

Tableau 2 : La segmentation des textes par les retours à la ligne

Nous pouvons observer que, dans une proportion non négligeable d'écrits, des alinéas appuient encore plus fortement la fonction démarcative déjà relevée du fait de la fréquence d'utilisation du point, comme dans cette copie :

« Il le suivit.

Une demi-heure plus tard Bambou s'arrêta.

Bob en profita pour le récupérer.

Il se rendit compte qu'il était perdu au milieu de la forêt.

Il prit Bambou dans ses bras. »

Ces usages des marques de ponctuation sont complétés par des connecteurs. Et (49,9\% des connecteurs au total) est l'organisateur textuel le plus employé, comme le montrent de nombreux travaux antérieurs (Favart \& Passerault, 1995 ; Mouchon, Fayol et Gombert, 1991 ; Jaffré, 1997). Quant aux connecteurs 
diversifiés utilisés, ils respectent les relations inhérentes à la structure narrative (Favart \& Passerault, 1999).

\begin{tabular}{|l|l|}
\hline Connecteurs & $\begin{array}{l}\text { Pourcentages par rapport au nombre total des } \\
\text { connecteurs }\end{array}$ \\
\hline ET & $49,9 \%$ \\
\hline MAIS & $16,7 \%$ \\
\hline ALORS & $10,2 \%$ \\
\hline PUIS & $8,7 \%$ \\
\hline QUAND & $6,9 \%$ \\
\hline CAR & $3,3 \%$ \\
\hline
\end{tabular}

Tableau 3 : La fréquence des connecteurs dans les copies observées

Nous avons noté que la segmentation des écrits analysés était opérée grâce à différents moyens linguistiques qui couvrent le large spectre du paradigme de la ponctuation.

\begin{tabular}{|l|l|l|}
\hline & Nombre & Pourcentages \\
\hline Propositions & 3293 & $100 \%$ \\
\hline Points (. ? !) & 1733 & $52,6 \%$ \\
\hline Virgules & 366 & $11 \%$ \\
\hline Connecteurs & 1339 & $40,6 \%$ \\
\hline
\end{tabular}

Tableau 4 : La répartition des marques interpropositionnelles dans les textes analysés

Les connecteurs marquent 40,6 \% des phrases graphiques. Ils sont parfois en redondance avec certains signes de ponctuation comme la virgule ou peuvent être employés seuls comme l'illustre cet extrait : «Il marcha et il trébucha sur une boîte il l'ouvrit et trouva un trésor et il continua à marcher à marcher jusqu'à ce qu'il trouve la sortie il retrouva ses parents et il retourna chez lui. » Nous retrouvons ici une illustration de la planification pas à pas de type knowledge telling (Bereiter \& Scardamalia, 1987). Cet extrait, qui montre un enchaînement d'actions reliées entre elles par le seul «et », atteste les difficultés cognitives qui peuvent encore persister en sixième lors de la production libre d'un texte narratif, empêchant la réalisation d'un produit cohérent et planifié globalement.

Ainsi, au niveau linguistique, nos résultats interrogent la notion de phrase (Béguelin, 2002). Aucun texte ne rend compte totalement de la «ponctuation zéro ». On peut dire que les compétences rédactionnelles des élèves illustrent la segmentation en phrases sur la voie de la normalisation. On peut trouver par exemple des guillemets qui marquent la fin d'une phrase à la place d'un point ( Quand Max se réveilla, il vit le mot et dit : si on l'ouvrait » «ouvrons-la »). Certains élèves observés expérimentent le spectre des marques qu'ils ont rencontrées au hasard de leur lecture. Une copie présente des points de suspension avec des parenthèses par exemple.

Grâce à une approche plus qualitative, nous avons également constaté une grande disparité des compétences démarcatives des élèves, ce qui pose le problème du traitement didactique de ces compétences par les enseignants. Tour à tour dans les écrits observés, le point, la virgule, l'alinéa, la ponctuation du dialogue, les connecteurs et même le mot FIN (qui ponctuent $62 \%$ des textes observés) ont une fonction démarcative et « ponctuent » les phrases. Cette diversité, même si elle n'est pas toujours normalisée, ne permet pas de parler de sous-ponctuation. Nous avons donc vu qu'une position 
linguistique ne suffisait pas pour rendre compte des compétences scripturales des élèves à un temps « $\mathrm{t}$ » de leur apprentissage.

Quel traitement didactique peut-on apporter aux difficultés des élèves recensées dans notre étude ? Comment dans les classes, peut-on enseigner la ponctuation? Que nous apprennent les pratiques effectives d'enseignement?

\section{Une réponse didactique aux besoins des élèves : enseigner la ponctuation en classe de sixième}

Les analyses de pratiques effectives d'enseignement apportent des réponses sur les objets enseignés en classe. Nous proposons pour ce colloque une étude de cas extraite de notre thèse dans laquelle nous avons interrogé les «enseignables » concernant la ponctuation. Nos travaux - l'étude que nous présentons ici étant selon nous caractéristique des suivis de professeurs opérés au cours de notre recherche doctorale montrent en effet que cet objet d'enseignement se laisse difficilement circonscrire et que l'enseignant doit multiplier les approches didactiques pour garantir les apprentissages de ses élèves. Les manuels scolaires sont de plus en plus pertinents sur la notion de ponctuation. Toutefois, les pistes didactiques les plus performantes apparaissent, selon nous, dans des ouvrages didactiques comme celui de Bessonnat \& Brissaud (2001) ou celui de Campana (2002) dans lesquels les activités didactiques tiennent compte des travaux des sciences d'appui que sont la linguistique et la psycholinguistique.

\subsection{Méthodologie}

Nous privilégions, dans cette étude, la méthodologie d'ingénierie didactique (Artigues, 2002) en procédant à une étude de cas ( Paolacci, 2005). Un scénario de séance extraite de Campana (2002) est proposé à un professeur de collège ( classe de sixième). La notion de phrase graphique y est opposée à la " phrase orale » en montrant que ce sont deux types de production langagière différents, autonomes et sans hiérarchisation possible. L'objectif des activités proposées est de "faire prendre conscience de la différence entre écrit et oral » (Campana, 2002 : 72).

Des étapes très précises de travail sont déclinées par Campana (2002: 72) :

- Le professeur donne à lire une transcription écrite d'un discours oral. L'exemple est un fait divers issu d'une émission radio. L'auteur propose un texte sans aucune marque de ponctuation et avec de nombreuses répétitions. Les réactions des élèves sont sollicitées.

- L'enseignant fait ensuite écouter l'extrait de l'émission radio. Les élèves entendent alors les pauses et les intonations de l'oral.

- Lors d'une deuxième écoute, la tâche des élèves est de noter par un trait vertical les pauses qu'ils perçoivent. Lors d'une mise en commun, une harmonisation des propositions de segmentation a lieu.

- Lors d'une troisième écoute, les élèves doivent être attentifs à la longueur des pauses et aux différents types d'intonation qui coïncident avec ces pauses : soit montantes, soit descendantes.

- On demande ensuite de recopier l'énoncé transcrit en plaçant la ponctuation et les majuscules «qui semblent correspondre aux deux types de pauses que les élèves ont identifiées ». Les phrases obtenues ne sont pas acceptables : on doit en conclure qu'il n'y a pas de correspondance entre pauses, intonation et ponctuation.

- La dernière étape de la séquence consiste à réécrire en groupes la transcription du récit oral de telle sorte qu'elle soit acceptable à l'écrit. Parmi les versions proposées, un texte est choisi et recopié par l'enseignant au tableau. Le maître lit à haute voix le texte « en demandant aux élèves de porter leur attention sur les liens entre intonations et pauses » (p. 73). Les élèves doivent avoir conscience que les pauses marquées sont celles de la lecture à haute voix. 
Comment le professeur observé s'empare-t-elle de ce contenu didactique prescriptif? Qu'en est-il du savoir effectivement enseigné en classe? Nous procédons, pour répondre à ces questions, à un recueil de données désormais classique en didactique du français (Schneuwly \& Thevenaz-Christen (éds), 2006). La séance de classes est filmée. Les données recueillies concernent également les supports donnés aux élèves et les écrits du professeur sur le tableau noir.

\subsection{Résultats et analyse}

La planification du professeur observé montre des choix qui se démarquent du scénario de Campana. Nous transcrivons ci-dessous les notes manuscrites du professeur.

\section{« La distinction oral/écrit}

Etape 1 : Texte transcrit donné aux élèves. Il s'agit de la retranscription du commentaire oral de Bastien, élève de la classe, à la suite de l'extrait de la traversée de la Mer Rouge du film Les dix commandements.

Etape 2 : Ecoute de la cassette

On comprend mieux : intonation, pauses. L'oral transcrit n'est pas de l'écrit.

Etape 3 : On relève les marques de l'oral

Etape 4 : On écoute la cassette et on marque les pauses. Elles correspondent aux intonations, à la ponctuation.

«Supprimez tout ce que vous n'utilisez pas à l'écrit. »

Etape 5 : Réécrire le texte pour qu'il soit communicable.

Que retenir? Ce qui différencie oral/écrit.

- La ponctuation guide le lecteur.

- Il n'y a pas de phrases à l'oral comme à l'écrit.

- Il est important de prendre en compte les critères de l'écrit pour être lu et compris. »

Notons que l'écrit original de l'enseignante est fortement raturé au niveau de l'étape $4:$ "on écoute la cassette et l'on marque des pauses. Elles correspondent à l'intonation et à la ponctuation ». L'hésitation du professeur au moment de sa planification a son importance au regard des enseignements qui vont suivre. Le professeur interprète la suggestion de Campana ainsi : une pause à l'oral équivaut à un signe de ponctuation, ce qui n'est pas tout à fait exact (d'après Campione \& Véronis, 2002). Campana, en se référant aux théories de référence, écrit même le contraire. Mais on doit reconnaître que la proposition de Campana de faire écrire des phrases incorrectes pour arriver à la conclusion que l'intonation, les pauses et la ponctuation ne coïncident pas, obéit à une préoccupation plus linguistique que didactique.

Qu'en est-il de l'objet effectivement enseigné ? La séance a lieu au mois de mars dans une classe de sixième et est consécutive à une séance de langue sur la grammaire de la phrase au cours de laquelle le professeur a abordé les notions de thème et propos. Le scénario général de la séance effectivement menée permet de mettre en relief les écarts entre les prévisions et la mise en œuvre effective.

Tableau 5 : La séance effectivement menée

\begin{tabular}{|c|l|l|l|}
\hline Etapes & Caractéristiques & Durée & Pourcentage \\
\hline 1. & Introduction des activités & $3 \mathrm{mn}$ & $6,7 \%$ \\
\hline 2. & Lecture de l'écrit de transcription & $3 \mathrm{mn}$ & $6,7 \%$ \\
\hline
\end{tabular}




\begin{tabular}{|c|l|l|l|}
\hline 3. & Commentaires sur cet écrit & $9 \mathrm{mn}$ & $20 \%$ \\
\hline 4. & Ecoute de la cassette & $2 \mathrm{mn}$ & $4,5 \%$ \\
\hline 5. & Réactions après l'écoute & $10 \mathrm{mn}$ & $22,2 \%$ \\
\hline 6. & $\begin{array}{l}\text { Repérages des marques de l'oral dans l'écrit de } \\
\text { transcription }\end{array}$ & $5 \mathrm{mn}$ & $11,1 \%$ \\
\hline 7. & Mise en commun et tableau « oral/écrit» & $13 \mathrm{mn}$ & $\begin{array}{l}28,8 \% \\
\text { durée totale : } \\
45 \mathrm{mn}\end{array}$ \\
\hline
\end{tabular}

L'écrit, support de l'activité, est une transcription d'un monologue oral d'un enfant de la classe (Bastien) commentant un extrait de film. Les élèves lisent la transcription qu'a effectuée l'enseignante. Aucune pause n'est notée. Voici un extrait de l'écrit distribué : « Il y a surtout des euh des effets spéciaux qui sont quand même surprenants oui euh la musique est quand même elle suit quand même beaucoup le film bon quand même c'est pas mal pour un film. »

Lors de l'étape 3 de la séance effective, Bastien a la parole le premier pour qualifier cet écrit : «c'est du charabia ». Le professeur note au tableau les remarques des élèves en les sélectionnant et les reformulant : « charabia; pas de signe de ponctuation; des hésitations, des répétitions ; on n'écrirait pas ainsi ; on improvise ; on a un interlocuteur présent qui réagit ; on ne réfléchit pas avant de parler; on se corrige ; intonation». Le professeur ajoute le problème oublié de la redondance du sujet («la musique elle accompagne le film »), sans noter celui des négations incomplètes. Pendant ces échanges ponctués par la prise de notes du professeur au tableau, les élèves n'écrivent pas. L'étape 5 est caractérisée par la consigne suivante: " vous allez reprendre le texte de Bastien et vous allez surligner tout ce que vous n'écririez pas + d'accord ? + donc vous soulignez ce que vous n'auriez pas à l'écrit ». Les élèves procèdent à une identification des procédés de l'oral. Le professeur remplit un tableau de deux colonnes « écrit/oral » sous la dictée des élèves en commençant par la colonne de l'oral : « euh, répétitions, voilà donc quand même ».

On peut dire que ces échanges entre le professeur et les élèves participent à la caractérisation du discours oral. Nous pouvons observer que l'objet effectivement enseigné diffère de l'objet initialement prévu. Certaines tâches prévues dans la planification du professeur sont écartées. Ainsi, la tâche de marquer les pauses après l'écoute de la cassette pour introduire la ponctuation n'est pas retenue délibérément, si l'on en croit cette remarque de l'enseignante à la classe : «on pourrait faire un exercice mais il ne me semble pas très intéressant on verra quand on le transformera à l'écrit ».

La modalité de l'écrit n'est envisagée que deux minutes avant la fin de la séance. La deuxième colonne du tableau de synthèse final est remplie hâtivement par le professeur. Chaque item concernant l'oral est mis face à un item caractérisant l'écrit :

\begin{tabular}{|l|l|}
\hline oral & écrit \\
\hline euh & Suppression \\
répétitions & un vocabulaire plus précis \\
voilà donc quand même & une ponctuation forte \\
\hline
\end{tabular}

La disposition de ce tableau illustre de notre point de vue les représentations du professeur concernant les différences « écrit/oral »: l'écrit découlerait de l'oral. Le système de flèches liant terme à terme l'oral et l'écrit peut être également considéré comme une préparation concrète au travail de réécriture qui va 
suivre. Le professeur argumente le dernier point du tableau en faisant allusion au rôle ponctuant des connecteurs: « on peut faire l'hypothèse que chaque fois qu'on a fini avec une idée + à l'oral il n'y a pas de ponctuation mais il y a un mot qui dit qu'on en a fini avec l'idée peut-être à l'écrit il faudrait en garder certains de ces petits mots mais en remplacer certains par une ponctuation forte donc on terminera làdessus ». Le professeur fait allusion, par ces propos, au modèle de Schneuwly (1988) et au problème de la segmentation textuelle.

Par manque de temps et par souci de tenir compte des interventions des élèves, les items de l'écrit sont en deçà de ce qu'avait prévu le professeur, qui avait anticipé les points suivants: «le texte suit un ordre ; il n'y a pas de répétition; les interventions personnelles de l'interlocuteur disparaissent; les négations sont complètes ; les phrases sont composées de leur thème et de leur propos ; la ponctuation guide le lecteur ».

Le professeur, par les tâches demandées aux élèves, invite sa classe à une réflexion métalinguistique sur le discours oral. Cet objet d'étude est assez peu abordé au collège. De proche en proche, les élèves sont amenés à prendre conscience que la ponctuation concerne exclusivement l'écrit. La difficulté de lecture de la transcription du monologue de Bastien est une conséquence de l'absence de phrases et de signes de ponctuation, ce que remarquent immédiatement les élèves. Implicitement, certaines caractéristiques de l'écrit sont donc mises en valeur. Par contre, contrairement à ce que Campana proposait, le travail sur la phrase n'a pas été réalisé. Le choix didactique du professeur a repoussé ce thème à la séance sur la réécriture de la transcription du discours oral qui a succédé. Cette réécriture est une tâche qui met en œuvre des compétences scripturales concernant directement les notions de phrase et de ponctuation. Les élèves, dégagés de la recherche des idées, se focalisent sur des compétences rédactionnelles dites de surface : le choix des mots, l'orthographe, la syntaxe et la ponctuation.

Le scénario de Campana (2002) illustre bien la nécessité de ponctuer un texte écrit pour être lisible et compris. Notre analyse a montré que l'objet enseigné dans la séance observée est autre, tout en restant dans la problématique générale posée par l'acte d'écriture. Le professeur observé, confronté, par le biais de notre méthodologie de recherche, à un thème qui lui était peu familier, a été déstabilisé ; ce qui a eu pour effet de creuser l'écart entre le prévu et le réalisé. D'autres études sont en cours et convergent vers le même constat : les enseignants sont mal à l'aise avec l'enseignement de notions linguistiques dont ils ignorent ou méconnaissent l'arrière-plan théorique (ce qui pose le problème du lien entre didactique et théories de référence ou de la formation des professeurs)

\section{En prolongement, former les enseignants à l'enseignement de la ponctuation}

Nous venons de voir, par la présentation de ces deux études ( l'analyse du corpus de copies d'élèves et des pratiques effectives d'enseignement ), deux problématiques présentes au cœur de la didactique du français illustrée par le volet « apprentissage » et le volet « enseignement ». Tous ces résultats prouvent que la ponctuation comme notion grammaticale scolaire est à la fois complexe à enseigner et à apprendre. Ceci nous a amenée, dans nos recherches doctorales, à une réflexion sur la formation à l'enseignement de la ponctuation qui préoccupent les chercheurs en IUFM que nous sommes. Les études concernant la question de la formation, et plus particulièrement la formation initiale, ont été menées dans le cadre de notre équipe de recherche l'Erte 46 du GRIDIFE ( Groupe de Recherche sur les Interactions Didactiques et la Formation des Enseignants) de l'IUFM de Midi-Pyrénées.

Dans un premier temps, citons une étude sur les pratiques déclarées des enseignants débutants (Paolacci $\&$ Garcia-Debanc, 2004). Pour cette étude, nous avons porté à notre analyse des travaux d'enseignants en deuxième année de formation (fiches de préparation et comptes rendus de séances a posteriori) élaborés pendant un stage. A partir des conclusions issues de cette recherche-prouvant que certaines représentations peuvent être un obstacle à une approche raisonnée de la notion de ponctuation - un module de formation a été conçu. 
Nous nous sommes centrées, par la suite, sur les pratiques effectives de formation ( Paolacci \& GarciaDebanc, 2005). Nos questions de recherche étaient les suivantes : Comment former des enseignants débutants à l'enseignement de la ponctuation ? Comment amener ces professeurs stagiaires à interroger leurs conceptions? Notre analyse a été basée sur des séances de formation dans les filières des professeurs d'école (PE) et des professeurs de collège et de lycée (PLC) à l'IUFM de Midi-Pyrénées.

Nous n'entrerons pas dans les détails des modules de formation dans cette communication. Retenons quelques principes. Avec les ateliers proposés, les professeurs stagiaires ont approché le paradigme des signes de ponctuation sur un plan linguistique mais aussi en référence aux savoirs en psycholinguistique. Ils ont lié de façon plus ou moins intuitive l'étude des emplois de la ponctuation avec les emplois des connecteurs comme et. Ils ont pu analyser les valeurs des signes de ponctuation (grâce à une analyse de la copie d'élève en particulier). Même si ces savoirs ne sont pas transposables à tous les problèmes de langue, les professeurs débutants ont pris conscience de la complexité de la ponctuation, « renfort de l'écriture », qui n'est «ni codé ni systématisé » (Béguelin, 2002) et ont été à même de mieux évaluer la nécessité d'une transposition didactique raisonnée pour enseigner tout objet linguistique. Les deux filières PE et PLC en formation initiale ne sont pas très éloignées au niveau de l'approche de l'enseignement/apprentissage de la langue comme le prouvent les données recueillies. On a souligné les mêmes difficultés à aborder la complexité d'une copie d'élève, les mêmes difficultés à traduire les conceptions des auteurs de manuels et aussi des interrogations similaires face au problème didactique que pose l'enseignement de la langue.

\section{Conclusion et perspectives}

Les intérêts de notre étude sont nombreux. La ponctuation, comme notion grammaticale, pose des difficultés à la fois pour l'enseignant et pour l'élève, comme nous l'avons montré. Notre thèse pose la question du pourquoi et propose des pistes pour répondre à cela. Il s'agit de modifier le regard souvent trop normatif des enseignants sur cet objet d'étude et c'est l'objectif que nous avons visé dans les études menées en formation initiale des enseignants.

A l'issue de nos travaux de thèse qui ont permis d'interroger les différents niveaux de reformulation des savoirs (Chevallard, 1991), nous avons mené une recherche sur la didactique de la phrase dans l'articulation école/collège (de 2004 à 2007). Avec une équipe de praticiens, nous avons exploré l'enseignement implicite de la grammaire par le biais des écritures-pastiches dont parlent les programmes officiels. Nous poursuivons, au moment où nous écrivons ces lignes, le suivi longitudinal d'un enseignant entrant dans le métier (Paolacci, 2007, pour une première approche) sur le thème de l'enseignement de la grammaire et plus spécifiquement de l'enseignement de la phrase. Nous tentons d'apprécier les effets à plus ou moins long terme de la formation à l'enseignement de la langue.

\section{Références bibliographiques}

Anis, J., Chiss, J.-L. \& Puech, C. (1988). L'écriture. Théories et descriptions. Bruxelles : De Boeck.

Artigues, M. (2002). Ingénierie didactique. Quel rôle dans la recherche didactique aujourd'hui. Les Dossiers des Sciences de l'Education. 8. 59-73.

Béguelin, M.-J. (2002). Clause, période ou autre ? La phrase graphique et la question des niveaux d'analyse. Y a-t-il une syntaxe au-delà de la phrase ? Verbum.1-2. . Tome XXIV. 85-109.

Béguelin, M.-J. (2000). De la phrase aux énoncés. Grammaire scolaire et descriptions linguistiques. Bruxelles : De Boeck Duculot.

Bereiter, C. \& Scardamalia, M. (1987). The Psychology of Written Composition. Hillsdale, NJ : Lawrence Erlbaum Associates.

Bessonnat, D. \& Brissaud, C. (2001). L'orthographe au collège. Paris: Delagrave.

Campana, M. (2002). Une grammaire pour mieux écrire. CRDP de Créteil. 
Campione, E. \& Véronis, J. (2002). Etude des relations entre pauses et ponctuations pour la synthèse de parole à partir de textes. Nancy : TALN.

Chevallard, Y.(1985, 1991). La transposition didactique. Grenoble : La pensée sauvage.

Catach, N. (Ed.) (1980). Langue française. 45.

Catach, N. (1994). La ponctuation. Paris : PUF.

Doppagne, A. (1978). La bonne ponctuation. Bruxelles : Duculot.

Drillon, J. (1991). Traité de la ponctuation française. Paris : Tel Gallimard.

Fabre, C. (1989). Les débuts de la ponctuation au CP. Etudes de linguistique appliquée. 73. 59-71.

Favart, M. \& Passerault, J.-M. (1995). Evolution du rôle fonctionnel des connecteurs et de la planification du récit écrit chez les enfants de 7 à 11 ans. Revue de Phonétique Appliquée. 115-116-117. 198-212.

Favart, M. \& Passerault, J.-M. (1999). Aspects textuels du fonctionnement et du développement des connecteurs: approche en production. L'Année Psychologique. 99. 149-173.

Favart, M. \& Passerault, J.-M. (2000). Aspects fonctionnels du point et de la virgule dans l'évolution de la planification du récit écrit. Enfance. 2. 187-205.

Fayol, M. (1981). L'organisation du récit écrit chez l'enfant. Son évolution de 6 à 10 ans. Thèse de Doctorat d'Etat. Bordeaux II.

Fayol, M. (1997). Des idées au texte. Psychologie cognitive de la production verbale, orale et écrite. Paris : PUF.

Garcia-Debanc,C.. \& Fayol, M. (2001). Apports et limites des modèles du processus rédactionnel pour la didactique de la production écrite. Dialogue entre psycholinguistiques et didacticiens. Pratiques. 115/116. 37-51.

Jaffré, J.-P. (1997). The Genesis of punctuation. In Conférence internationale The teaching and learning of punctuation. University of Manchester.

Mouchon, S., Fayol, M. et Gombert, J.-E. (1991). L'utilisation de quelques connecteurs dans les récits d'enfants de 5 à 8 ans. L'année psychologique. 89. 513-529.

Paolacci, V. (2005). Didactique de la ponctuation en production écrite dans la liaison école/collège. Thèse non publiée dirigée par C. Garcia-Debanc. Toulouse II Le Mirail.

Paolacci, V. (2007). Enseigner la grammaire du français langue maternelle. Qu'est-ce qui se joue dans la classe à l'école élémentaire française ? In Actes du colloque (CDROM). Didcog-didactique cognitive. Toulouse, septembre 2007.

Paolacci, V. \& Garcia-Debanc, C. (2004). Quel enseignement de la ponctuation ( et autres marques d'organisation textuelle) en formation initiale d'enseignants ? Repères. 27. 93-117.

Paolacci, V. \& Garcia-Debanc, C. (2005). Comment former à l'enseignement de la ponctuation? Analyse de pratiques effectives de formation initiale. Pratiques. 125-126. 85-114.

Passerault, J.-M. (1991). La ponctuation. Recherches en psychologie du langage. Pratiques. 70. 85-107.

Programmes de l'école élémentaire (2007). Paris : Scéren.

Programmes de français de la classe de sixième (1995). Paris : Scéren.

Schneuwly, B. (1988). Le langage écrit de l'enfant. Lausanne : Delachaux Niestlé.

Schneuwly B., Thevenaz-Christen T. (éds.) (2006). Analyses des objets enseignés. Le cas du français. Bruxelles : De Boeck.

Tournier, C. (1980). L'histoire des idées sur la ponctuation, des débuts de l'imprimerie à nos jours. Langue française. 45. 28-41.

Védénina, L.-G. (1989). Pertinence linguistique de la présentation typographique. Paris : Peeters. 